\title{
Análise Econômica de Sistemas de Produção a Pasto para Bovinos no Município de Campos dos Goytacazes-RJ'
}

\author{
Afonso Aurélio de Carvalho Peres ${ }^{2}$, Paulo Marcelo de Souza ${ }^{3}$, Hernan Maldonado 4 , \\ José Fernando Coelho da Silva ${ }^{5}$, Cezar da Silva Soares ${ }^{6}$, Suzana Correa Wagner Barros ${ }^{7}$, \\ Ismail Ramalho Haddade ${ }^{8}$
}

\begin{abstract}
RESUMO - Objetivou-se, com este trabalho, analisar a viabilidade econômica de diferentes sistemas de recria de fêmeas em pastagens de capim-elefante na região Norte Fluminense. Fez-se uma análise econômica de três sistemas de recria de novilhas mestiças leiteiras em pastejo de capim-elefante. A fim de se estudar o risco que cada sistema possa apresentar diante das oscilações de preços no mercado, utilizou-se a Simulação de Monte Carlo. Os sistemas estudados foram: sem suplementação (SP1), oferta de 0,5\% do peso vivo de concentrado (SP2), e pastejo alternado de capim-elefante com estilosantes (SP3). A análise foi realizada a partir da utilização dos indicadores econômicos Valor Presente Líquido (VPL) e Taxa Interna de Retorno (TIR). O VPL calculado, a uma taxa anual de desconto de $10 \%$, foi de $\mathrm{R} \$ 6.091,86, \mathrm{R} \$ 6.364,54$ e $\mathrm{R} \$ 215,43$, enquanto o capital investido apresentou TIR anual de 29,58; 30,10 e 10,46\%, respectivamente, para os três sistemas estudados. Utilizou-se a Análise de Sensibilidade para determinação dos itens de maior influência sobre a rentabilidade dos sistemas. Observou-se que os itens com maior impacto sobre o resultado econômico dos sistemas foram, em ordem de importância, o preço de venda das novilhas, o preço de aquisição das novilhas e o custo da mão-de-obra. Concluiu-se que o sistema SP3 apresentou maior risco econômico.
\end{abstract}

Palavras-chave: análise de sensibilidade, capim-elefante, simulação de Monte Carlo, suplementação, taxa interna de retorno, valor presente líquido

\section{Economical Analysis of Pasture Production Systems to Cattle in the Region of Campos dos Goytacazes-RJ}

\begin{abstract}
Different economic viability of pasture production systems to heifers grazing elephantgrass pasture in North Fluminense region wa analyzed. The economical analysis of three systems of growing fedlot dairy heifers grazing elephantgrass pasture was performed. In order to study the hazard that each system can present due to the oscillations of prices in the market, Monte Carlo simulation was used. The systems studied were: without supplementation (PS1), offer of $0.5 \%$ of the alive weight of concentrate (PS2), and alternate pasture of elephantgrass and Stylosanthes (PS3). The analysis was based on the economical indicators Net Present Value (NPV) and Internal Rates of Return (IRR). The NPV calculated at an annual rate of discount of $10 \%$, were $\mathrm{R} \$ 6,091.86, \mathrm{R} \$ 6,364.54$ and $\mathrm{R} \$ 215.43$, while the invested capital showed yearly IRR of $29.58,30.10$ and $10.46 \%$, respectively for three systems studies. The sensitivity analysis was used to identify the items of larger economical impact and that presented great effect on the profitability of the systems. In order of importance, the sale price of heifers, the purchase price of heifers and the labor cost were the items with greater impact on the economical results. It was concluded that the PS3 showed the highest economical stroke.
\end{abstract}

Key Words: elephantgrass, internal rates of return, Monte Carlo simulations, net present value, sensibility analysis, supplementation

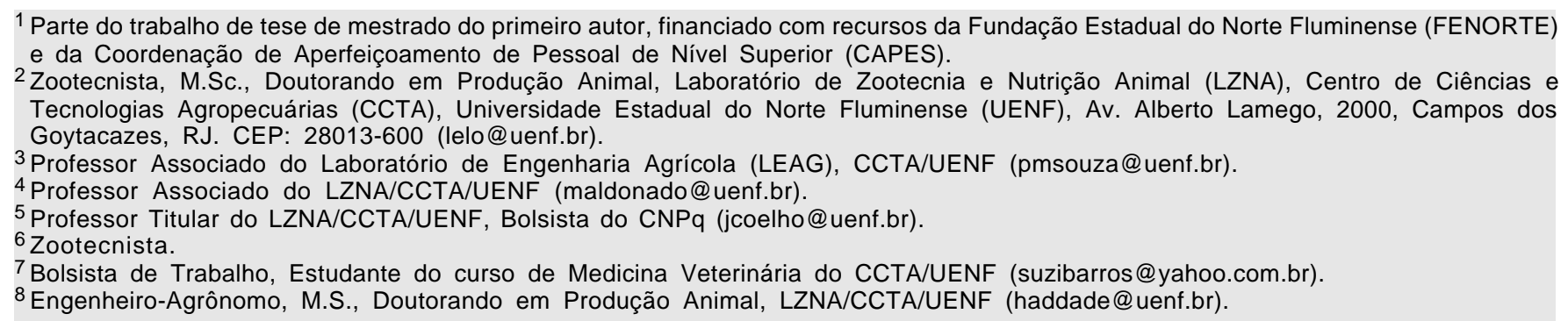




\section{Introdução}

Atualmente, qualquer atividade do setor agrícola, para se manter competitiva, deve ser avaliada continuamente sob o âmbito econômico. Os custos de produção da atividade, a receita obtida e a rentabilidade do capital investido são fatores importantes para o sucesso de qualquer sistema de produção. Esta análise permite a detecção do item que, em determinado momento, pode inviabilizar a atividade, como as oscilações de preços no mercado. Sabe-se que a intensificação dos sistemas de produção a pasto e a adoção de novas tecnologias podem contribuir para aumentar a disponibilidade do produto final (leite) e a conseqüente queda nos custos de produção (Matos, 1996). Entretanto, na literatura nacional, existem poucos trabalhos avaliando a viabilidade econômica de sistemas de produção em pasto para bovinos, o que dificulta tomadas de decisão pelo produtor rural.

Quando se trata da bovinocultura de leite, o desafio é maior, pois existem diversos sistemas de produção, em razão dos diferentes aspectos edáficos, climáticos, estruturais, composição do rebanho e, principalmente, do nível tecnológico em uso.

O desempenho econômico de um sistema de produção de machos e fêmeas Holandês-Zebu, na fase de recria, durante a estação seca, foi avaliado por Mendonça et al. (2000), que encontraram um lucro negativo, refletindo na remuneração parcial do capital investido. Brito et al. (2002) estudaram o fornecimento de suplementação a pasto para manutenção do peso de matrizes Canchim, durante o inverno. A análise econômica dos suplementos fornecidos foi realizada sob dois pontos de vista: produção de carne - em que os suplementos apresentaram rentabilidade negativa, ou seja, não foi possível remunerar o capital investido - e produção de tourinhos, em que a rentabilidade foi positiva, sendo encontrada taxa de remuneração mensal sobre o capital investido em torno de $2,5 \%$ ao mês. Essa taxa pode ser considerada satisfatória, uma vez que, no mesmo período, outra aplicação financeira disponível no mercado, a caderneta de poupança, apresentou taxas mensais de, aproximadamente, $0,60 \%$.

Alguns indicadores econômicos podem ser adotados para a avaliação financeira de sistemas de produção, entre eles o valor presente líquido (VPL) e a taxa interna de retorno (TIR). O VPL é considerado um critério de avaliação de projetos mais rigoroso e isento de falhas técnicas (Noronha, 1987;
Contador, 1988). Corresponde à soma algébrica dos valores do fluxo de caixa de um projeto, atualizados à taxa ou às taxas de desconto do período em questão. Segundo esse indicador, um projeto é viável se apresentar um VPL positivo. Na implantação do melhor projeto, escolher-se-á aquele que apresentar o maior VPL positivo. A taxa interna de retorno (TIR) é definida por Contador (1988) como a taxa de juros que iguala a zero o VPL de um projeto, ou seja, é a taxa de desconto que iguala o valor presente dos benefícios de um projeto ao valor presente de seus custos. Um projeto é viável e deve ser adotado quando sua TIR é igual ou maior que o custo de oportunidade dos recursos para sua implantação.

A análise de sensibilidade permite observar de que maneira as variações ocorridas em uma das variáveis dos sistemas podem influenciar sua viabilidade econômica, sendo possível determinar em que medida um erro ou modificação de uma das variáveis incide nos resultados do projeto (Buarque, 1991). Conhecer as variáveis com maior peso na determinação dos resultados de cada sistema é de extrema importância, pois, ao identificar os itens que exercem maior impacto econômico, pode evitar erros na tomada de decisão dos produtores e que venham proporcionar grandes consequiências ou até prejuízos na instalação do sistema escolhido.

Avaliar o risco econômico envolvido no sistema de produção é considerado de extrema importância, pois permite ao produtor planejar e executar o seu sistema de maneira que não venha ter insucesso na atividade. Para avaliação desse risco, a técnica da simulação de Monte Carlo vem sendo empregada. Exemplos de utilização dessa técnica para a abordagem do risco em atividades agrícolas podem ser encontrados em diversos trabalhos, como os de Almeida et al. (1985), Shirota et al. (1987), Noronha e Latapia (1988), Biserra (1994) e Araújo e Marques (1997). Segundo Noronha (1987), a simulação de Monte Carlo é, dentre os métodos que utilizam probabilidade na análise dos riscos, o mais simples e prático, além de apresentar custo razoavelmente baixo. Dada a impossibilidade de se estudar a distribuição de probabilidade de todas as variáveis componentes nos sistemas, a melhor alternativa consistiu em identificar, pela análise de sensibilidade, os componentes que apresentaram maior efeito sobre o resultado financeiro de cada sistema avaliado e, a partir daí, realizar a simulação de Monte Carlo. Adotar a distribuição triangular, que consiste em definir o 
preços médio, mínimo e máximo do item analisado, ocorrido durante um período determinado, pode auxiliar na execução da técnica de simulação. Tal distribuição é de extrema importância quando não se dispõe de conhecimento suficiente sobre o comportamento dos preços das variáveis, na região.

O presente trabalho foi realizado com o objetivo de verificar a viabilidade econômica de três diferentes sistemas de recria de novilhas de leite mestiças em pastagem de capim-elefante, testados na região Norte Fluminense.

\section{Material e Métodos}

O trabalho foi conduzido na Unidade de Pesquisas em Zootecnia da Universidade Estadual do Norte Fluminense (UENF), localizada no município de Campos dos Goytacazes, Estado do Rio de Janeiro. Foram avaliados três sistemas de recria de novilhas leiteiras mestiças Holandês-Zebu em pastagens de capim-elefante: 1. Sem suplementação (SP1); 2. Oferta de $0,5 \%$ do peso vivo de concentrado (milho em grão, $81,3 \%$ e farelo de soja, $18,7 \%$ ), ao final do dia em cocho (SP2) e; 3. Pastejo alternado, com três dias em capimelefante e três dias em estilosantes, cv. Mineirão (SP3). Forneceu-se suplementação mineral no cocho, ad libitum aos animais em todos os tratamentos.

As informações necessárias para a elaboração deste trabalho e construção dos fluxos de caixa para cada sistema testado a campo, bem como os dados utilizados (preços, vida útil etc) foram coletados junto aos produtores rurais, técnicos de extensão rural e estabelecimentos comerciais da região. Fez-se levantamento de todos os itens necessários para a implantação e manutenção de cada sistema, como mão-deobra disponível, máquinas e equipamentos utilizados, cercas, animais, adubos, entre outros. Os índices zootécnicos e o desempenho obtido pelas novilhas em cada sistema de produção foram utilizados para auxiliar na construção dos fluxos de caixa e determinar o tempo (ciclo) que cada grupo de animais permaneceria no sistema.

O fluxo de caixa foi construído para um período de 12 anos, compondo-se de entradas e saídas computadas mensalmente, uma vez que este período é suficiente para que seja esgotada a vida útil das cercas utilizadas. Durante este período, considerou-se uma reforma total das pastagens utilizadas, logo após seis anos de utilização. Cabe ressaltar que o dispêndio de capital para a reforma das pastagens

R. Bras. Zootec., v.33, n.6, p.1557-1563, 2004 pode ser evitado, se ocorrer bom manejo das pastagens, tornando-a auto-sustentável, durante o período considerado para a construção dos fluxos de caixas dos sistemas. Para auxiliar na construção dos fluxos de caixa, considerou-se que as novilhas utilizadas foram adquiridas aos 12 meses de idade, pesando em torno de $190 \mathrm{~kg}$ de peso vivo, compradas na região, ao preço médio de $\mathrm{R} \$ 300,00$. No período de fevereiro a dezembro do ano de 2001, foram utilizadas 22 novilhas leiteiras mestiças, sendo 12 animais "testers" e 10 animais "put and take", sendo alocadas quatro novilhas em cada sistema de produção. Foram obtidos ganhos médios diários (GMD) de 429, 624 e 535 g/animal, respectivamente, para SP1, SP2 e SP3. A partir desses ganhos, foi determinado o tempo que cada novilha permaneceria no seu respectivo sistema até atingir o peso vivo de $320 \mathrm{~kg}$, recomendado à primeira cobertura, sendo em seguida, inseminada e vendida ao preço médio de $\mathrm{R} \$ 1.000,00$.

$\mathrm{Na}$ construção dos fluxos de caixa, foram consideradas, como entradas, as receitas advindas da venda das novilhas e, como saídas, as despesas incorridas em aquisição das novilhas, aluguel da terra, benfeitorias (cochos, bebedouros e comedouros), operações mecanizadas terceirizadas (aração, gradagem, sulcação etc), mão-de-obra (campeiro, empreitada, inseminador e veterinário), insumos (adubos, calcários, mudas, sementes, herbicidas etc), alimentação (sal comum, sal mineral, milho e soja), medicamentos (vacinas, vermífugos, repelentes, remédios etc) e outras (análise de solo, brincos de identificação, sêmen bovino etc). Não foram considerados os custos com impostos e os gastos com a energia elétrica utilizada nas cercas, em função da dificuldade de mensuração destes gastos na área experimental.

Utilizaram-se, para efeito de estudo da análise econômica, dois indicadores econômicos: o VPL (valor presente líquido) e a TIR (taxa interna de retorno). A expressão para cálculo do VPL é a seguinte:

$$
V P L=\sum_{t=0}^{n} V F /(1+r)^{t}
$$

em que VPL = valor presente líquido; VF = valor do fluxo líquido (diferença entre entradas e saídas); $\mathrm{n}=$ número de fluxos; $r=$ taxa de desconto $; \mathrm{t}=$ período de análise $(i=1,2,3 \ldots)$.

No cálculo do VPL, aplicaram-se três taxas de 
desconto sobre o fluxo líquido mensal de cada sistema de produção. As taxas adotadas foram 8,10 e $12 \%$ ao ano.

Para a TIR, segundo os critérios de aceitação, quanto maior for o resultado obtido no projeto, maior será a atratividade para sua implantação. Assim, a TIR é o valor de r que iguala a zero a expressão:

$$
V P L=V F_{0}+\frac{V F_{1}}{(1+r)^{1}}+\frac{V F_{2}}{(1+r)^{2}}+\frac{V F_{3}}{(1+r)^{3}}+\cdots+\frac{V F_{n}}{(1+r)^{n}}
$$

em que VF = fluxos de caixa líquido $(0,1,2,3, \ldots, n)$; $\mathrm{r}=$ taxa de desconto.

Foi realizada a análise de sensibilidade para cada sistema de produção, visando observar as variações ocorridas nas variáveis componentes, avaliando a participação de cada item na viabilidade econômica do sistema testado. Para realização da análise de sensibilidade, os itens que compõem os fluxos de caixa foram estudados individualmente, buscando a real participação de cada item no sistema e o impacto proporcionado. Considerou-se variação de $1 \%$, sempre no sentido desfavorável para os resultados do sistema, nos preços de cada item do fluxo de caixa. Então, foi possível observar o item que apresentou maior efeito sobre os indicadores de resultado econômico do sistema, isto é, o VPL e a TIR.

De posse das informações obtidas nas análises anteriores, foi empregada a técnica da simulação de Monte Carlo para se avaliar o risco envolvido nos sistemas testados. No trabalho, adotou-se a distribuição triangular, considerando para cada item admitido como probabilístico, os preços mínimos, médios e máximos, registrados na região Norte Fluminense, durante o período experimental, no ano de 2001. Os preços foram coletados junto aos estabelecimentos comerciais da região. A partir destas definições, foram gerados pelo programa "MS-Excel", vários números aleatórios, sendo obtidos diferentes valores para as variáveis analisadas, resultando em vários fluxos de caixa e, conseqüentemente, diversos indicadores de resultados para cada sistema de produção. Pela repetição desse procedimento um número significativo de vezes, gerou-se a distribuição de frequiências do indicador do sistema, que permitiu aferir a probabilidade de sucesso ou insucesso do mesmo. Foram submetidas ao processo de simulação, as seguintes variáveis, identificadas na análise de sensibilidade: preço de venda das novilhas, preço de aquisição das novilhas, mão-de-obra contratada, mão- de-obra especializada, preço da terra, adubos (sulfato de amônio, superfosfato simples, cloreto de potássio e FTE), operações mecanizadas, milho em grão, farelo de soja, sal mineral e sêmen bovino. A partir daí, obteve-se o risco de cada sistema, frente às oscilações de preços.

\section{Resultados e Discussão}

Na Tabela 1, são apresentados os resultados do valor presente líquido (VPL) obtidos para cada sistema de produção, calculados a partir das taxas de desconto de 8,10 e $12 \%$ ao ano. Para os três sistemas de produção, o VPL foi positivo, com exceção para SP3, que não se mostrou viável à taxa de $12 \%$ a.a. Esta situação se deve ao aumento dos gastos com a mão-de-obra utilizada para a limpeza e manutenção dos pastos formados pela leguminosa, elevando os custos do sistema.

A relação entre o VPL e o custo de oportunidade do capital dos sistemas de produção mostra que, quanto maior a taxa de desconto aplicada, menos atrativo se torna o sistema. Cabe ressaltar que esta é uma situação experimental que tende a apresentar custos unitários elevados, visto que os custos de produção por animal tendem a diminuir com a expansão do empreendimento para escalas mais apropriadas. De qualquer modo, a ocorrência de valores econômicos que tenham sido super ou subestimados afeta igualmente os três sistemas de produção, de forma que a comparação dos indicadores de rentabilidade obtidos para cada um permanece válida. À taxa de desconto de $10 \%$, os três sistemas de produção testados foram viáveis economicamente, por ordem de importância, SP2, SP1 e SP3.

Utilizando o indicador econômico taxa interna de retorno (TIR), obteve-se, respectivamente, 29,58; 30,10 e $10,46 \%$ ao ano, para SP1, SP2 e SP3. As taxas encontradas foram favoráveis à adoção de qualquer sistema, pois foram superiores às remunerações obtidas em investimentos disponíveis no mercado, como a caderneta de poupança, por exemplo, cuja remuneração situou-se em torno de $6 \%$ ao ano, no mesmo período.

A resposta de cada sistema de produção a uma variação desfavorável de $1 \%$ nos preços de cada item componente do fluxo de caixa encontra-se na Tabela 2.

Para os três sistemas de produção, observa-se que o indicador de rentabilidade (VPL) é mais sensível à variação de preço de venda das novilhas. $\mathrm{O}$ item preço de compra foi considerado o segundo de maior

R. Bras. Zootec., v.33, n.6, p.1557-1563, 2004 
Tabela 1 - Valor presente líquido (em $R \$$ ) dos sistemas de produção um, dois e três, considerando taxas de desconto de 8,10 e $12 \%$ ao ano

Table 1 - Net present value (R\$) of the production systems one, two and three, considering yearly discount rates of 8,10 e $12 \%$

\begin{tabular}{cccc}
\hline \multicolumn{4}{c}{$\begin{array}{c}\text { Valor presente líquido }(\mathrm{R} \$) \\
\text { Net present value }(R \$)\end{array}$} \\
\hline & \multicolumn{3}{c}{$\begin{array}{c}\text { Taxa de desconto }(\%) \\
\text { Discount rate (\%) }\end{array}$} \\
\cline { 2 - 4 } $\begin{array}{c}\text { Sistemas de produção } \\
\text { Production systems }\end{array}$ & 8 & 10 & 12 \\
\hline SP1 & $7.447,14$ & $6.091,86$ & $4.959,68$ \\
SP2 & $7.759,10$ & $6.364,54$ & $5.198,60$ \\
SP3 & $1.264,25$ & 215,43 & $-648,03$ \\
\hline
\end{tabular}

impacto sobre a viabilidade econômica para SP1 e SP2, enquanto para SP3 foi a mão-de-obra. Cabe ressaltar que, no sistema de produção SP2, os itens milho e soja apresentaram grande influência sobre o VPL, sendo considerados importantes, por envolverem fontes de alimentos essenciais na formulação da ração. Um exemplo da variação de preços destes alimentos está no valor constatado em março de 2001 para a saca de soja e milho $(50 \mathrm{~kg})$, vendidos na região Norte Fluminense a, respectivamente, $\mathrm{R} \$ 34,80$ e $\mathrm{R} \$ 16,50$ ou US\$14.75 e US\$6.99 (US\$1.00 = R \$2,36). Ao término do ano, a saca de soja chegou a $\mathrm{R} \$ 41,00$ (US\$17.37) e a do milho manteve-se estável.

Ao adotar o sistema de produção SP2 ou SP3, o produtor rural, pode-se ter aumento de até $20 \%$ nos preços de venda das novilhas, porque a adoção desses sistemas permitiu aos animais atingir o peso de cobertura em menor tempo, em virtude do melhor ganho de peso vivo diário apresentado. Diante dos índices adotados e da idade dos animais, observou-se que seriam necessários, aproximadamente, 360 dias para que as novilhas permanecessem no SP1 até atingirem a idade a primeira cobertura, ao passo que, nos sistemas SP2 e SP3, seriam necessários, 270 e 315 dias, respectivamente. Ao antecipar a idade à primeira cobertura, conseqüentemente, esses animais serão vendidos nos meses compreendidos no período seco do ano, quando o preço de venda das novilhas passa de $\mathrm{R} \$ 1.000,00$ (US \$423.73) para R $\$ 1.200,00$ (US \$508.47) na região. Embora esse possível aumento de preços não tenha sido considerado na construção dos fluxos de caixa, esta observação é importante, uma vez que a venda dos animais em períodos de maior valor implicaria em aumento nas receitas dos sistemas SP2 e SP3, tornando mais atraentes seus indicadores econômicos de viabilidade.

Na Tabela 3, são apresentados os resultados da análise probabilística do risco para cada sistema de produção estudado. Pode-se observar que o sistema SP3 apresentou o maior risco de se tornar inviável, com taxas de 70,19 e 78,67\%, quando submetido às taxas de desconto de 10 e $12 \%$, respectivamente. O sistema SP1 teve retorno mais baixo do capital investido, pois o ciclo de recria foi maior, porém o risco de se tornar inviável foi menor. Esse fato é atribuído ao menor dispêndio de capital com a contratação de mão-de-obra e compra de fontes alimentares utilizadas como suplementação, cuja variação de preços implica em elevação dos riscos.

A Figura 1 ilustra os vários VPLs foram simulados na análise de Monte Carlo. Pode-se observar que SP1 apresenta o menor risco quando o VPL do sistema é igual a zero, ao passo que SP3, o maior risco.

Tabela 3 - Resultado da análise probabilística do risco: probabilidade dos sistemas de produção apresentarem VPL negativo, considerando taxas de desconto de 10 e $12 \%$ ao ano

Table 3 - The risk profitability analysis result: the production systems profitability were negative NPV considering yearly discount rates of 10 and $12 \%$

\begin{tabular}{lrr}
\hline & \multicolumn{2}{c}{$\begin{array}{c}\text { Taxa de desconto (\%) } \\
\text { Discount rate }(\%)\end{array}$} \\
\cline { 2 - 3 } $\begin{array}{l}\text { Sistemas de produção } \\
\text { Production systems }\end{array}$ & 10 & 12 \\
\hline SP1 & 5,08 & 7,75 \\
SP2 & 11,70 & 15,17 \\
SP3 & 70,19 & 78,67 \\
\hline
\end{tabular}


Tabela 2 - Resultado da análise de sensibilidade: mudança em reais do VPL, devido a uma variação, no sentido desfavorável, de $1 \%$ nos preços

Table 2 - Result of the sensitivity analysis: percentual change in NPV, due to a variation, in the unfavorable sense, of $1 \%$ in the price

\begin{tabular}{|c|c|c|}
\hline \multicolumn{3}{|c|}{$\begin{array}{c}\text { Análise de sensibilidade } \\
\text { Sensitivity analysis }\end{array}$} \\
\hline Sistemas & Categoria & Variação do VPL $(\mathrm{R} \$)$ \\
\hline Systems & Category & $N P V$ variation $(R \$)$ \\
\hline \multirow{11}{*}{ SP 1} & Venda de novilhas (unidade) & 261,05 \\
\hline & Compra de novilhas (unidade) & 85,46 \\
\hline & Mão-de-obra não qualificada (homem/dia) & 49,01 \\
\hline & Adubo sulfato de amônia a $20 \%$ (saco de $50 \mathrm{~kg}$ ) & 11,85 \\
\hline & Preço de terra (hectare) & 10,93 \\
\hline & Adubo cloreto de potássio a $60 \%$ (saco de $50 \mathrm{~kg}$ ) & 7,55 \\
\hline & Operações mecanizadas (trator/hora) & 5,59 \\
\hline & Sêmen bovino (dose) & 5,26 \\
\hline & Adubo superfosfato simples a $20 \%$ (saco de $50 \mathrm{~kg}$ ) & 4,92 \\
\hline & Veterinário para detecção de prenhez (dia) & 3,52 \\
\hline & Sal mineral ( saco de $25^{5} \mathrm{~kg}$ ) & 3,27 \\
\hline \multirow{13}{*}{ SP 2} & Venda de novilhas (unidade) & 352,29 \\
\hline & Compra de novilhas (unidade) & 112,62 \\
\hline & Mão-de-obra não qualificada (homem/dia) & 49,01 \\
\hline & Milho em grão (saco de $50 \mathrm{~kg}$ ) & 32,72 \\
\hline & Farelo de soja tostado (saco de $50 \mathrm{~kg}$ ) & 17,27 \\
\hline & Adubo sulfato de amônia a $20 \%$ (saco de $50 \mathrm{~kg}$ ) & 13,19 \\
\hline & Preço de terra (hectare) & 10,93 \\
\hline & Adubo superfosfato simples a $20 \%$ (saco de $50 \mathrm{~kg}$ ) & 8,34 \\
\hline & Adubo cloreto de potássio a $60 \%$ (saco de $50 \mathrm{~kg}$ ) & 8,03 \\
\hline & Sêmen bovino (dose) & 7,10 \\
\hline & Operações mecanizadas (trator/hora) & 5,58 \\
\hline & Veterinário para detecção de prenhez (dia) & 4,75 \\
\hline & Sal mineral (saco de $25 \mathrm{~kg}$ ) & 3,10 \\
\hline \multirow{12}{*}{ SP 3} & Venda de novilhas (unidade) & 309,24 \\
\hline & Mão-de-obra não qualificada (homem/dia) & 120,22 \\
\hline & Compra de novilhas (unidade) & 99,65 \\
\hline & Preço de terra (hectare) & 21,72 \\
\hline & Adubo superfosfato simples a $20 \%$ (saco de $50 \mathrm{~kg}$ ) & 11,92 \\
\hline & Operações mecanizadas (trator/hora) & 9,48 \\
\hline & Adubo sulfato de amônia a $20 \%$ (saco de $50 \mathrm{~kg}$ ) & 6,47 \\
\hline & Sêmen bovino (dose) & 6,23 \\
\hline & Adubo cloreto de potássio a $60 \%$ (saco de $50 \mathrm{~kg}$ ) & 4,19 \\
\hline & Veterinário para detecção de prenhez (dia) & 4,17 \\
\hline & Adubo micronutrientes FTE (saco de $50 \mathrm{~kg}$ ) & 3,24 \\
\hline & Sal mineral (saco de $25 \mathrm{~kg}$ ) & 3,21 \\
\hline
\end{tabular}




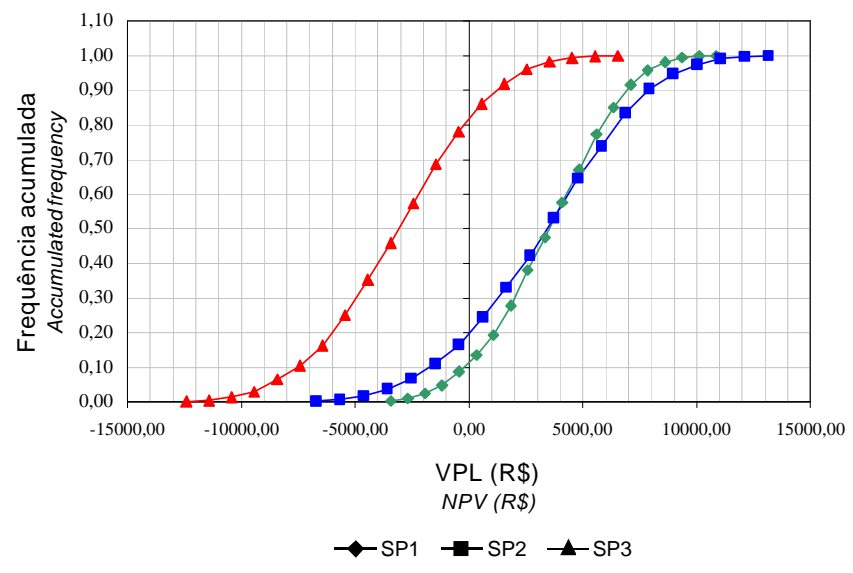

Figura 1 - Freqüência acumulada dos valores presentes líquidos simulados para os sistemas de produção SP1, SP2 e SP3.

Figure 1 - Accumulated frequency of simulations net present values to production systems PS1, PS2 and PS3.

\section{Conclusões}

Os três sistemas propostos de recria de novilhas leiteiras mestiças em pastagens de capim-elefante, para a região Norte Fluminense, foram economicamente viáveis.

O maior valor presente líquido e a melhor taxa interna de retorno foram obtidos no sistema de produção em pastagem de capim-elefante com fornecimento de suplementação concentrada (SP2).

Os itens preço de venda das novilhas, preço de aquisição das novilhas e custo da mão-de-obra foram identificados como os de maior impacto sobre a viabilidade econômica.

Por ordem de importância, o sistema de produção que utilizou somente pastagem de capim-elefante apresentou o menor risco de investimento, frente às mudanças de preços, ocorridas no mercado, seguido pelo sistema com suplementação concentrada e pelo sistema com leguminosa.

\section{Agradecimento}

À Fundação Estadual do Norte Fluminense (FENORTE) e à Coordenação de Aperfeiçoamento de Pessoal de Nível Superior(CAPES), pelo apoio finaceiro.

Às gerências da FENORTE, pelos serviços prestados.

\section{Literatura Citada}

ALMEIDA, L.A.S.B.; VIEIRA, M.C.; GASPARINO FILHO, J. et al. Factibilidade econômica sob condições de riscos: análise de moinhos de milho para suplementação de farinha de trigo. Revista de Economia e Sociologia Rural, v.23, n.2, art. 3, CD-ROM, 1985.

ARAÚJO, M.P.; MARQUES, P.V. Rentabilidade, em condições de incerteza, na produção avícola sob contratos de integração vertical em Minas Gerais. Revista de Economia e Sociologia Rural, v.35, n.3, p.23-43, 1997.

BISERRA, J.V. Rentabilidade da irrigação pública no Nordeste, sob condições de risco: o caso do perímetro Morada Nova. Revista de Economia e Sociologia Rural, v.32, n.3, art. 4, CD-ROM, 1994.

BRITO, R.M.; SAMPAIO, A.A.M.; CRUZ, G.M. et al. Limitação do requerimento microbiano em energia metabolizável fermentável ou proteína degradável na suplementação do pasto de inverno de vacas da raça Canchim. Revista Brasileira de Zootecnia, v.31, n.1, p.112-118, 2002.

BUARQUE, C. A incerteza para seleção de projetos. In: BUARQUE, C. (Ed.) Avaliação econômica de projetos. 8.ed. Rio de Janeiro: Campus, 1991. p.179-196.

CONTADOR, C.R. Indicadores para seleção de projetos. In: CONTADOR, C. (Ed.) Avaliação social de projetos. 2.ed. São Paulo: Atlas, 1988. p.41-58.

MATOS, L.L. Perspectivas em alimentação e manejo de vacas em lactação. [M.G.]: Centro Nacional de Pesquisa de Gado de Leite, 1996. 5 p. (Comunicado técnico)

MENDONÇA, S.S.; CAMPOS, J.M.S.; ASSIS, A.J. et al. Sistema de produção de recria de machos e fêmeas leiteiras a pasto. II. Desempenho econômico na estação seca. In: REUNIÃO ANUAL DA SOCIEDADE BRASILEIRA DE ZOOTECNIA, 37., 2000, Viçosa, MG. Anais... São Paulo: Gmosis, 2000. CD-ROM. Pôster. Sistema de produção e economia, art. 0937.

NORONHA, J.F. Projetos agropecuários: administração financeira, orçamento e viabilidade econômica. 2.ed. São Paulo: Atlas, 1987. 269p.

NORONHA, J.F.; LATAPIA, M.X.L.C. Custos de produção agrícola sob condições de risco no Estado de São Paulo. Revista de Economia e Sociologia Rural, v.26, n.3, art. 2, CD-ROM, 1988.

SHIROTA, R.; SILVA, R.D.M.; LIMA, R.A.S. et al. Técnica de simulação aplicada a avaliação econômica de matriz de duas linhagens de frango de corte. Revista de Economia e Sociologia Rural, v.25, n.1, art. 5, CD-ROM, 1987. 